\title{
Solving the 'Two Times Problem' With an Information Gathering and Utilizing System (IGUS)
}

\author{
Ronald P. Gruber ${ }^{1}$ \\ Carlos Montemayor ${ }^{2}$ \\ Richard A. Block ${ }^{3}$
}

1. School of Medicine, Stanford University rgruber@stanford.edu

2. Dept of Philosophy, San Francisco State University cmontema@sfsu.edu

3. Dept. of Psychology, Montana State University block@montana.edu

*Correspondence: rgruber@stanford.edu 


\begin{abstract}
There is a long standing 'two times problem' in that a satisfactory reconciliation between the time of physics and that of psychology has not been realized. A partial solution to the past/present/future phenomenon has been successfully given by the Hartle information gathering and processing system (IGUS) view. That model IGUS robot is enhanced here for the entire 'two times problem' to deal with not only the temporal experiences of the flow of time but also those of manifest time. A dualistic robot is proposed which has a veridical system of temporal experiences that are compatible with various spacetime cosmologies. It also has an illusory system of corresponding temporal experiences. This dualistic IGUS robot was made possible by discovering temporal experience within the brain that correspond to those of physics. The dualistic theory suggests that the veridical system, as a result of evolution, begets the illusory system to enhance behavioral adaptation. Thus, there is just one fundamental physical time which the brain does, indeed, possess and then enhances with illusory counterparts. Therefore, there should no longer be a need to reify illusory temporal experiences as modern spacetime cosmologies tend to do. Physical time already resides within human time.
\end{abstract}

Key words:1. IGUS, 2. flow of time, 3. passage, 


\section{Introduction}

\subsection{Two times problem.}

There is a long standing debate regarding the nature of time and the need to reconcile the time of physics with the time of human experiences. Much of the discussion centers around the 'frozen' Block Universe of Einstein which is favored by many if not most physicists [1]. Also, there is the view from some physicists that time does not exist at a fundamental level but is derive[2,3] cosmology that provides a mechanism to account for the 'flow' that the brain undeniably experiences. For example, Ellis [4] (see also [5]) proposed a universe that grows, the edge of which provides the passage (flow) that humans experience. Dowker [6] utilizes a spacetime Causal Set Theory which is based upon a suggested growth of spacetime atoms in a non-physical mathematical construct. She says it "breathes life" (p.22) into the Block Universe by providing the physical occurrence of an event. Smolin [7] has gotten around the problem by simply declaring that time is real, motion is real, we should not ignore our senses, and the past does disappear.

Perhaps the best and most succinct summation of the 'two times problem' was provided when James Gleick [8] reported on the physicist Feynman's view on the illusion of time.

"It seemed to Feynman that a robust conception of 'now' ought not to depend on murky notions of mentalism. The minds of humans are manifestations of physical law, too, he pointed out. Whatever hidden brain machinery created (one's) coming into being must have to do with a correlation between events in two regions of space - the one inside the cranium and the other elsewhere 'on the spacetime diagram."

On Richard Feynman, 1963 This was a concise phrase to contrast and relate human time to physical time. Feynman was asking for a physical explanation for the human experience of time. He felt that there must be a fundamental connection between the two times and that it would ultimately involve physics.

\subsection{Introducing the IGUS}

Gell-Mann and Hartle [9] introduced the notion of an information gathering and utilizing system (IGUS) for a quasiclassical domain to help explain the consistent histories formalism, the mystery of why we continue to experience a quasi-classical world. They argued that certain types of IGUS -such as ourselves- 'evolve to exploit' the regularities in some particular quasiclassical domain: "The one reason such systems as IGUSes exist, functioning in such a fashion, is to be sought in their evolution in the universe. Subsequently, Hartle [10] utilized the IGUS model to deal with Einstein's “stubborn illusion" of the past/present/future. His explicit stated goal was to recover the division between past, present and future. He emphasized that the world is four-dimensional according to fundamental physics, governed by basic laws that operate in a spacetime that has no unique division into space and time. Yet our subjective experience is divided into present, past, and future. He went on to discuss the origin of this division in terms of simple models of information gathering and utilizing systems $\sim$ IGUSs. His 
claim was that past, present, and future are not properties of four-dimensional spacetime, but notions describing how individual IGUSs process information. Their origin is to be found in how these IGUSs evolved or were constructed. The present, moreover, is not a moment of time in the sense of a spacelike surface in spacetime. Rather, there is a localized notion of present at each point along an IGUS's world line.

\subsection{The IGUS in detail}

A simple schematic for the human (model) IGUS is given in Fig. 1. At every proper time interval $t^{*}$, the robot captures an image of its external environment. In this case, the robot experiences a stack of cards labeled $a, b, c, d, e, f$, etc. whose top member changes from time to time. The IGUS robot chooses how to route and utilize its information. The robot uses the images in registers $P_{0}, P_{1}, P_{2}$ and $P_{3}$ in two processes of computation: $\mathrm{C}$ (conscious), and $\mathrm{U}$ (unconscious). The process $U$ uses the data in all registers to update a simplified model or schema of the external environment. A schema of the external environment is used by $\mathrm{C}$ together with the most recently acquired data in $P_{0}$ to make predictions about its environment to the future of the data in $P_{\mathrm{0}}$, make decisions, and direct behavior.

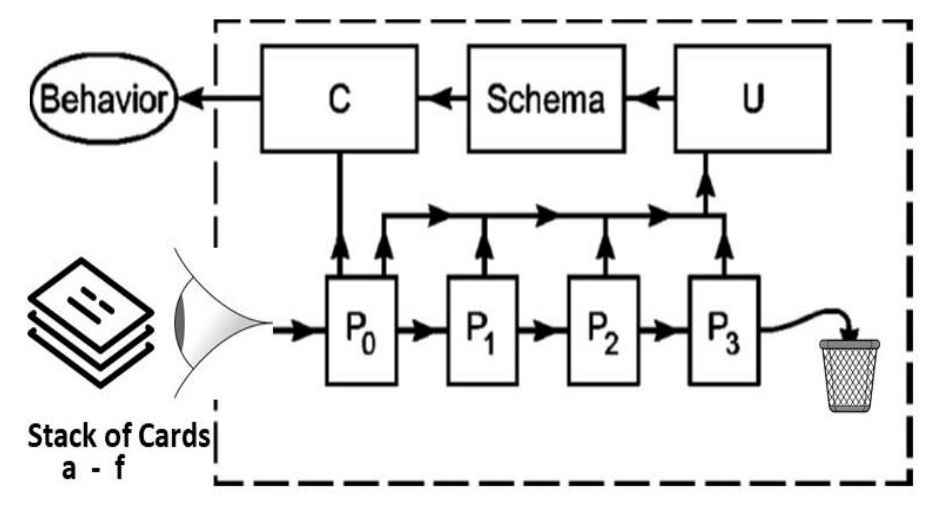

Fig. 1 Information gathering and utilization system (IGUS) of Hartle

In brief, the Hartle view allows one to appreciate the subjectivity of the moving present ('now') that is also compatible with accepted physical laws. Regarding the actual 'flow' of time (FOT) he attributes that to the movement of information in and out of the $\mathrm{C}$ (consciousness) register. In other words, the experiential flow component of the FOT is attributed to the utilizing system of the robot and not the time of physics. 'Flow' for the IGUS is not a property of physical time.

Hartle proposed a falsification test for his hypothesis. He suggested it should be possible to construct IGUS robots that process information differently and therefore experience different 'presents.' For example, a robot with a split visual system (SS robot) could experience the present with one half screen and events from the immediate past with the other half screen. It would confirm that there is no unique 'present.' A human is said to be one such robot (a 'model IGUS') in the sense that its 'present' is not a unique moment in spacetime. Rather, there is a 'present' at each instant along the robot's worldline. If that is the case the 'present' is subjective 
and, therefore, the notion of a unique, moving present (a present that moves) is an illusion. The majority of physicists agree. More recently, for example, Romero [11]discusses this view of the 'present' or 'now' and concurs that there is no physical flow of time, only an ordered system of events.

\subsection{Confirming the IGUS hypothesis}

To experimentally confirm the hypothesis that the present is not unique Gruber and Smith [12]chose to test Hartle's IGUS hypothesis that a new 'present' can be fabricated suggesting that the current one we humans possess is not unique. It involved the construction of a split screen $(S S)$ robot using a VR headset screen containing two 'presents' of slightly different local time intervals. However, upon construction, it was immediately apparent that the observer did not experience two simultaneous 'presents' because there was no sense of immersion in the environment. To create this immersion experience, a similar robot was created in which the observer is permitted to alternate between 'past' and 'present' screens ad libitum (the intermittently behind, IB robot). By being able to switch between equally realistic time periods, the observer experienced what was intended in the split screen $(S S)$ robot except in an alternating instead of a simultaneous manner. The participant was also allowed to go back and forth between 'past' and 'present' ad libitum by pressing a button. Unsolicited participant comments, that it clearly felt like 'being in the past,' were received. A few participants even indicated that they sometimes 'got lost' between what was 'past' and what was 'present.'

A worldline description of the 'present' for an $I B$ robot is given in Fig. 2. It shows the worldline of an external object that is the source of its images such as a stack of cards. This source changes its shape at discrete instants of time delineated by ticks, passing through configurations $\mathrm{c}, \mathrm{d}, \mathrm{e}, \mathrm{f}$, and g. As an example, the object $\mathrm{E}$ is recorded as e in two adjacent registers - thus e,e. The number of registers for e is simply proportional to the duration of observation. The image in each register is then experienced as $C_{e}$. These e's are experienced again as $C_{e}$ when each e moves to another register that is further away (along the world line). In short, the robot is permitted to utilize information as it chooses. In this case the present from register $e$ is experienced at two different points along the worldline, i.e., the IB robot experiences the same present twice. Thus, the 'present' is not necessarily unique. 


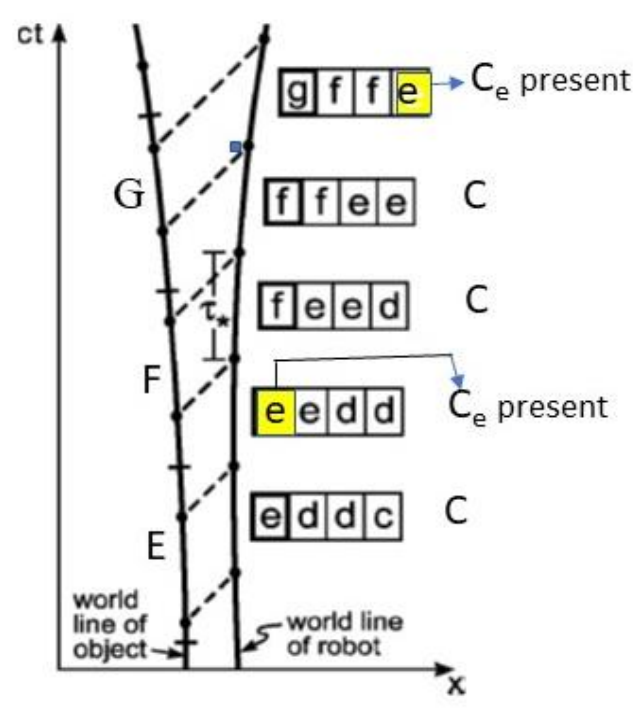

Fig. 2 A worldline description of the 'present' for an $I B$ robot. There are two 'presents' and therefore not unique.

Verifying Hartle's prediction suggested that the brain has as one of its fundamental temporal experiences an experience of past/present/future with a unique present. To be clear, although it was possible to construct a robot other than the 'model robot,' the IB robot does not prove that the 'moving present' is an illusion. It only establishes that there is a 'present' at each point along the worldline. The actual 'moving present' is a dynamistic illusory experience that is more related if not identical to the experience of 'moving,' in other words, motion which is described as an illusion below.

\section{Evolution of the IGUS model}

That IGUS concept to deal with the 'two times problem' was picked up by Jenann Ismael [13, 14]. She extended it to include flow and higher-level temporal beliefs - her 'supercharged IGUS.' A few others $[15,16,17]$ share the view as to the importance of IGUS to explain passage (flow). Recently, Callender [18, p.227] provided a most needed expansion of the model IGUS and relied upon it for the "beginnings of a theory of time flow." He augmented the IGUS robot with "gadgets" to account for many other phenomena, including objective temporal experiences all of which come under the heading of what he calls 'manifest time' which comprises essentially all temporal experiences. Each 'gadget' is a temporal experience needed to bring the robot closer to its human counterpart.

Gruber et al [19] expanded the IGUS concept further by introducing a "dualistic model' of temporal experiences. It was postulated that each of the components of the flow of time (FOT), including the past/present/future phenomenon have both illusory and non-illusory (veridical i.e., real) aspects. It was demonstrated that the brain did, indeed, contain temporal experiences that were congruent with those belonging to various spacetime cosmologies. For example, sequential order has as its counterpart temporality (the before/after experience). That IGUS model was 
derived in part from 10 chosen spacetime cosmologies (see Table I of ref. 19). Many of the spacetime cosmological claims take Einstein's Block Universe as a basis. Quantum mechanics has also led to the many modifications. Notable is the view that objects in the universe are really events that 'happen.' In other words, the Block is not frozen. Corresponding to those veridical experiences the brain also contains illusory counterparts. All the illusory experiences of the FOT were then contrasted to what these spacetime cosmologies have to say about those very same phenomena. Then it was possible to construct a dualistic model. Without a doubt there would be controversy here simply because the 10 spacetime views are not unanimous and it was necessary to decide which ones will most likely be correct and sustained.

Recently, that dualistic model, which included the IGUS robot was expanded further in the form of a unified theory for all the major temporal experiences [20]. The IGUS robot was used as a basis upon which an even wider variety of 'gadgets' were added to account for all of the temporal experiences of what is now referred to as 'manifest time.' Fig. 3 contains a list of the major physical and illusory parameters. As before, the assertion made was that the mind is dualistic in that there is a veridical system incorporating temporal experiences for physical time and a corresponding illusory system of temporal experiences. It was proposed that the illusory system is a product of evolution to augment the veridical system for the benefit of human adaptation.

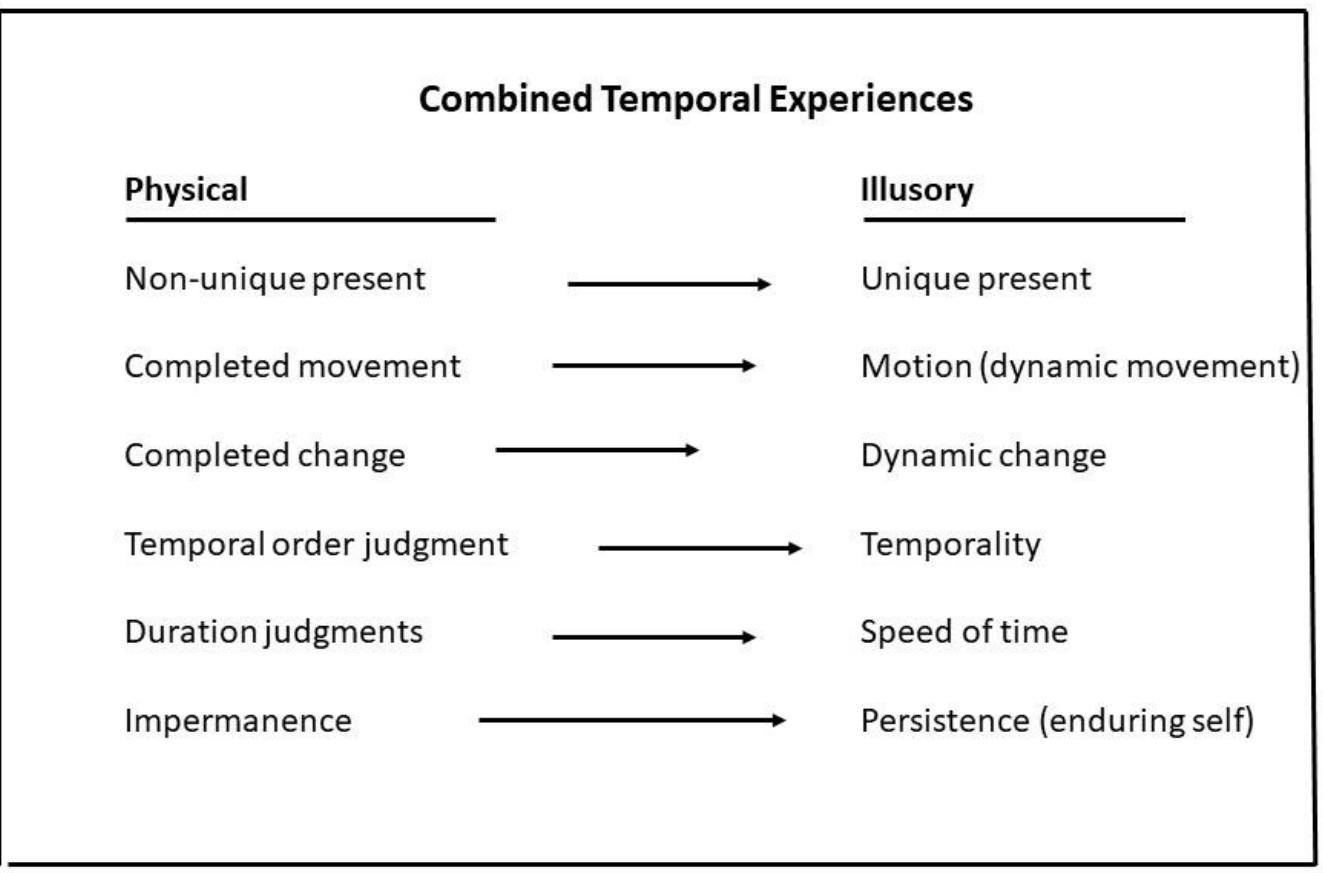

Fig. 3 Major physical and corresponding illusory temporal experiences of the brain

\section{3 'Gadgets' for the dualistic IGUS}

\subsection{Change (Completed and Dynamic)}


Physicists have tried to account for 'change' in their cosmological theories and psychologists have been interested in it for completely different reasons. Now it is possible to recognize the veridical and illusory aspects of change in order to reconcile them when viewed as a dualistic component of manifest time. It all begins with Rensick [21] who provided a seminal study on the dualistic nature of change. There is 'dynamic change' - the experience of seeing an illusory change occur such as one color or shape to another. There is also 'completed change' - the nonillusory experience that the change 'must have occurred.' The former experience is evoked by the brain when the frequency of stimuli (with a blank interstimulus interval) is relatively high. But when it is low the experience of completed change is evoked in which the subject must admit "I did not see the change occur but I know it must have happened." The difference between the two is that dynamic change is illusory and completed change is real (veridical). The brain evokes the dynamism of change to fill the gap between stimuli.

Of importance, 'completed change' within the dualistic IGUS is congruent with the change of accepted spacetime cosmologies. For example, despite the fact that he suggests we forget time Rovelli [22 p.97] takes the position that change is 'real.' Therefore, of necessity, the dualistic IGUS should possess that particular veridical component of manifest time, and it does, namely 'completed change.' The veridicality of change is also endorsed by Aerts [23] in his theory of 'Refounding Relativity' where he provides a method to account for the dynamism of change. Important is the fact that the dualistic IGUS has both and satisfies physics and psychology. Fig. 4 indicates that completed change is one of the physical temporal experiences that the brain does possess, and is added to the IGUS robot.

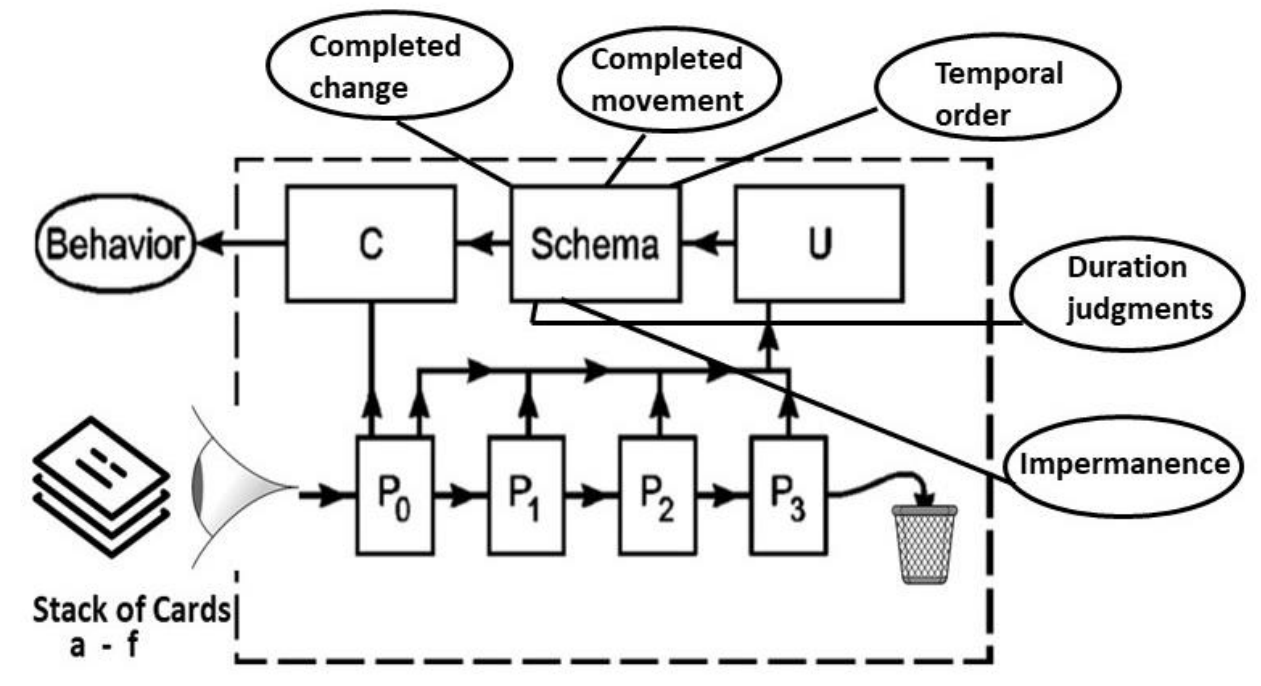

\section{Physical Time Within Human Time}

Fig. 4 The IGUS 'robot' with physical parameters of time added

\subsection{Completed Movement and Motion (Dynamic Movement)}


In neuroscience, the experience of motion is considered to be an illusory percept for the following reason. Visual perception is generally said to be discrete at a rate of $10-13 \mathrm{~Hz}$. This was determined by the classical continuous wagon wheel illusion. For details see references [24] and [25]. Assuming that perception is discrete Koch [26 p.274] suggests that motion is "painted on" to each perceptual frame. Although not immediately evident, motion (also termed dynamic movement) is the illusory counterpart of a (veridical) completed movement. An experimental example of it is in the form positional change as given by Nakashima and Yokosawa [27 p. 269] in a flicker change detection task. Just as in the completed change experiment above, completed movement was demonstrated at low stimulus frequency whereas dynamic movement (motion) was only seen at a relatively high frequency.

A question arises: how much of human observational experience is involved with completed movement? Although no surveys have been done it should be apparent that much of the time a person does in deed look away before returning back to view again. In the process of so doing there is a lack of continuity such that the motion (dynamic movement) will not be experienced. On the other hand, it is not bothersome to be denied dynamicity for all visual observation. Needless to say, motion is denied in the Block Universe, 'End of Time' and the 'Order of Time' views. Physical continuity is not necessarily in the cosmological schemes. Instead, what is expected by them is that events, including cerebral events, be discrete. Therefore, we add motion/completed movement as a 'gadget' to the dualistic IGUS.

\subsection{Temporal Order Judgments and Temporality}

Related but very different from the temporal experience of movement are the phenomena of human temporality (the before/after experience) and also (sequential) order from physics. For a review see Ruhnau [28] and Montemayor \& Wittmann [29]. For example, whereas there is order (asymmetry) to the events of the universe (Big Bang at one end) there is also a dynamic temporality for those events that the brain evokes and experiences [30]. The illusory nature of the temporality experience was brought to the fore by Arstila [31] by analyzing the phenomenon of succession. He notes that "the succession of experiences and the experience of succession are two different things." Here, it can be said that the former is a (veridical) temporal order judgment [32] whereas the latter is the experience of temporality. Of note, the information acquired by an observer is not different between temporal order judgment and temporality. To some degree, therefore, the additional experience of 'before/after' (the experience of succession) would appear to be illusory or at least outside of physics. Moreover, these two experiences (temporal order judgment and temporality) are separate experiences. If the interstimulus interval between auditory stimuli of different frequencies is reduced below a certain minimum, e.g., 10-15 msec, the observer experiences non-simultaneity, i.e., there is a temporality experience of before/after, but which tone came first cannot be ascertained. Having now claimed that the brain has the ability to experience both the illusory and the veridical aspect of order, a gadget of temporality/temporality order judgment is added to the dualistic IGUS.

\subsection{Duration Judgments and Speed of Time}

Another example of a veridical temporal experience is duration judgment. Duration judgment has been studied thoroughly with its prospective and retrospective types $[33,34]$. Prospective 
duration judgment involves some sort of timing mechanism. An actual 'internal clock' has not been discovered but 'population clocks' are a possible answer [35]. Retrospective duration judgment involves memory, and in particular the memory and contextual cues of those events, all of which provide objective duration measurements even if not necessarily accurate.

The corresponding illusory experience for duration judgment is 'speed of time,' i.e., the experience associated with 'how fast time' went. Droit-Volet and Wearden [36] studied this phenomenon referring to it as the 'passage of time judgments.' However, because the term passage is used differently by cosmologists the term 'speed' is used here. This temporal experience is assessed by asking the participants to indicate how quickly time seemed to pass during a task which is different than its duration. With respect to the two times problem there is no substantial importance here except that it completes the view that every veridical temporal experience has an illusory counterpart.

\subsection{Impermanence and Persistence (Enduring Self)}

A human needs to feel that she persists and is not simply a conglomerate of impermanent (ephemeral) events as spacetime cosmologies suggest. The observer, in a unique (moving) present, wants to believe she is a single persisting individual (an 'enduring self') and not multiple momentary individuals extending backwards in time. However, in the Block Universe, persistence of that sort has no place. When consoling the wife of his best friend Besso who had just died, Einstein said that Besso was still there. The implication was clear: there is a Besso who may be dead but another who is alive in the past. A theoretical time machine [37] would help settle that issue. Clearly, this time illusion is difficult to accept for most. However, it is much easier to acknowledge impermanence as veridical if one happens to hold the spacetime view that the universe is composed of events, and that the observer, too, is basically a series of complex events $[11,22]$.

To help confirm that persistence is an illusory experience a pilot experiment demonstrated that it can be precluded [19]. Observers ('human IB robots') wearing the backwards-in-time VR apparatus were allowed to watch a remote controlled toy dog roaming about as they went 'back and forth in time.' They lost the experience of persistence. Going back and forth into the past (e.g., $60 \mathrm{sec}$ back) she would note a moving toy dog. When she was in the past, she might see the dog to her right even though it is actually located to her left. When she returned suddenly (in a fraction of a second) to the present she would see the dog to her left where it actually is. The experience is that the dog does not appear to be the same dog because it could not have traveled several feet that quickly. The explanation is based upon the principle of spatiotemporal priority which occurs for the well-studied phenomenon of 'object persistence' [38]. When deciding whether an object is the same persisting object from some earlier time, factors relating to how and where that object has moved will almost always trump factors relating to what the object looks like.

Surprisingly there are some who opt for the view that the 'self' need not be persistent or at least should choose not to be persistent. Outside of Western civilization there are those who subscribe to that view. It is a belief amongst those adhering to Buddhism [39]. It is a belief that the individual is really, or at least should consider herself to be 'ephemeral' (a fleeting self). Their 
belief is that this illusion is the root of the suffering inherent in the human condition. Therefore, Buddhism takes the approach that its followers should ("for their own good") inhibit the illusion of the 'self.' That their view coincides with the veridical view of physics regarding impermanence (ephemerality) is undoubtedly a coincidence. Now that the duality of impermanence and persistence is established it can be added as yet another 'gadget' to Hartle's IGUS model robot.

\section{Physics' attempt to reify temporal illusions}

The modern 'two times problem' began with Einstein's Block Universe (BU), and it was quickly realized that physics just killed time by having a 'frozen' BU. Einstein, himself, was bothered by the 'stubborn illusion' of past/present/future. Ever since, physicists have tried to resuscitate time so that human time would not be an illusion. There has been an understandable need to legitimize the illusory time of humans. Declaring time is dead and what we experience is an illusion leaves us in an awkward place. Hartle's IGUS robot was the first successful attempt to deal with that illusion by demonstrating that the past/present/future experience is generated by IGUSs, and that it is "consistent with four dimensional spacetime physics" [10 p.101]. This view can be interpreted that the past/present/future experience is outside of physics much the way music is, and therefore not an illusion, i.e., not specifically contradicted by physics. As for the experience of FOT he attributes it to the movement of information into the $\mathrm{C}$ register. By so doing there is said to be a physical (neurophysiological) basis for it and therefore, it is taken out of the illusory category, also.

Many other physicists with various spacetime cosmological views have also tried to find reality within the human temporal experiences of time. Ten illustrative cases are reviewed in detail elsewhere [19]. For example, Ellis [4] introduced a growing BU the peripheral aspect of which is said to provide the human experience of the FOT. Elitzur's [5] Spacetime Dynamics Theory shares a similar view. Dowker [6 p. 22] utilizes the Casual Set Theory to "breathe life" into the BU. That theory is based upon a suggested growth of spacetime atoms in a non-physical mathematical construct to provide the physical occurrence of an event. Aerts $[23,40]$ has a spacetime cosmological view in which he insists with good arguments that change is the one time parameter that must be real. For him, 'coming into existence' is said to be the flow in the FOT. Muller $[41,42]$ also accounts for the human temporal experiences such as motion with his 'Now' cosmological theory. It involves a '4D model of progressing time,' i.e., space and also time are said to be continually created and 'real.' After providing a spacetime cosmological view that emphasizes order, Rovelli [22 p.197] concludes that the growth of entropy can account for the passage of time.

A detailed example of this attempt to reification is seen in Barbour's theory [2]. His timeless cosmology is achieved by starting with the timeless Wheeler-DeWitt equation,

$$
\hat{H}(x)|\psi\rangle=0
$$

which describes a static situation: the 'wave function of the universe.' The solution of the above equation gives relative probabilities for each possible static relative configuration space of the complete universe. Each configuration is associated with an instant of experienced time. Each 'snapshot' upon quantum wave reduction provides information about the event at that moment and also memories of prior snapshots which provide a direction to the nows (to the succession of 
snapshots). When needed, the conventional notion of Newtonian time can be recovered from this timeless picture and written as:

$$
\sum_{\mathrm{i}<\mathrm{j}} \mathrm{m}_{i} \mathrm{~m}_{\mathrm{j}} / \mathrm{r}_{\mathrm{i} j}=\sum_{i} \mathrm{~m}_{\mathrm{i}}\left(\mathrm{dx}_{\mathrm{i}} / \mathrm{d} \lambda\right)^{2}
$$

Important here is the claim that physical time is secondary. But, then a recent modification in the theory was proposed in which time might actually have two directions emanating from the 'Janus Point' [43]. But, more importantly for our purposes, the human temporal experience of time is said to be due to the arrow of complexity [44]. And, since complexity is physical and not illusory, human time is said to be given a veridical (real) basis.

What is apparent by all these different physical approaches is that there is a long standing movement to reify human time. However, it is substantially veridical by the dualistic IGUS view. Human time has a non-illusory temporal system that should satisfy those who have been uncomfortable believing that the gist of human temporal experiences is illusory. Quite the contrary, human time has a complete veridical system, parts of which have been ignored and parts of which have only been recently discovered. Therefore, the illusory system does not have to be shunned. The past/present/future illusion and all the other illusory temporal experiences should be viewed as welcome additions to the corresponding veridical temporal experiences that exist. Physical time within the cranium is alive and well. There is no need for 'breathe life' into the BU in order to justify the illusory temporal experience. That does not mean that the various proposed modifications of the BU are not valid. For example, the expanding BU may quite possibly be valid independently of the illusory temporal experience of passage and its components such as motion and temporality. Thus, it can be safely said now that the veridical system within the cranium is the basic temporal system for human adaptation. The illusory system merely augments and supplements it.

\section{Conclusions}

The Hartle IGUS model has been upgraded by others and now by our extended version. It is suggested that 'gadgets' representing components of manifest time, including the components of flow (passage) of time be added to the IGUS, and that these 'gadgets' are dualistic. It is noted that the brain happens to have a set of veridical experiences that are congruent with the views of modern spacetime cosmology. It is also noted that all the veridical experiences have corresponding illusory experiences. As a result of natural selection, the veridical system begot the illusory system in order for the human to be more behaviorally adaptive. The dualistic mind approach avoids implicating all human experiences of time as illusory and also removes the compulsion to insist that the 'flow' is real or veridical. By the compromise approach here that both 'times' exist and should co-exist, the two times problem is offered a very specific solution.

\section{Acknowledgments}

We would like to thank James Hartle, Julian Barbour, James Kalamas and Ryan P. Smith for helpful discussions of this complex topic. Also, many thanks to David Karp and Stephen Waddell for their technical expertise. 
No funding received for this project.

\section{References}

1.Davies, P. That mysterious flow. Scientific American, pp. 40-47 (September)2002

2.Barbour, J. "The emergence of time and its arrow from timelessness" in Physical Origins of Time Asymmetry, eds. J. Halliwell et al, (Cambridge University Press, Cambridge 1994.

3.Rovelli, C. Forget time Foundations of Physics, 2011,41:1475.

4.Ellis, G. The evolving block universe and the meshing together of times.

Annals of the New York Academy of Sciences, 1326:26-41.2014

5.Elitzur, A. Consciousness and the passage of time: Two persistent wonders-or one? Frontier Perspectives, 1992, 2: 27-33.

6.Dowker, F. The birth of spacetime atoms as the passage of time. Annals of the New York Academy of Sciences,2014, 1326: 18-25.

7.Smolin, L. Time Reborn. Boston; Houglton, Mifflin \& Harcourt.2013

8.Gleick, J. Genius. the life and science of Richard Feynman. New York: Open Road Media.2011

9.Gell-Mann, Murray,; James B. Hartle. "Equivalent sets of histories and multiple quasiclassical realms." arXiv preprint gr-qc/9404013 (1994).

10.Hartle J. The physics of now. American Journal of Physics, 2005,73:101-9.

11.Romero, G. Present time. Foundations of Science. 2015,20:135-145.

12.Gruber, R.P.; Smith.R.. An Experimental Information Gathering and Utilization Systems (IGUS) Robot to Demonstrate the Physics of Now. American. Journal of Physics. 2019,87:3019.

13.Ismael, J. On Whether the Atemporal Conception of the World Is Also Amodal Analytic Philosophy, 2015,56:142-157. 
14.Ismael, J. Passage, Flow, and the Logic of Temporal Perspectives." In Time of Nature and the Nature of Time. Boston Studies in the Philosophy and History of Science, vol 326 eds. Christoph Bouton and Philippe Huneman, 23-38. Cham, Springer.2017

15.Dorato, M.; Wittmann,M. The phenomenology and cognitive neuroscience of experienced temporality. Phenomenology and the Cognitive Sciences, 2019,1326:18-25.

16.Huggett, N. Reading the past in the present. In Time's arrow and the probability structure of the world eds. Barry Loewer, Brad Westlake and Eric Winsberg.

Cambridge: Harvard University Press.2018

17.Hertzel, M. Making sense of temporal passage. Intuitions,2016,13:35-48

18.Callender, C. What makes time special? Oxford: Oxford University Press.2017

19.Gruber, R P.; Montemayor, C.; Block, R. A. From Physical Time to a Dualistic Model of Human Time. Foundations of Science, 2020,25,927-54

20.Gruber, R.P., Montemayor, C., and Block, R.A. The physical time within human time: a new (IGUS robot) theory of manifest time. 2021 Submitted to arXiv.

21.Rensink, R. Change detection. Annual. Review of. Psychology,2002, 53:245-277.

22.Rovelli, C. The order of time. New York, Riverhead Books.2018

23.Aerts, D. (2018). Relativity theory refounded. Found Sci 2018,23:511-547.

.24.VanRullen, R.; Koch. C. Is perception discrete or continuous? Trends in Cognitive Sciences, 2003,7:207-13.

25.VanRullen, R.; Reddy, L.; Koch, C. A motion involved illusion reveals the discrete nature of visual awareness. In Space and time in perception and action. eds. R. Nihawan and B. Khurana. New York: Cambridge University Press. 2010

26.Koch, C. The quest for consciousness. Englewood: Roberts.2004

27.Nakashima R.; Yokosawa, K. Sustained attention can create an (illusory) experience of seeing dynamic change. Visual Cognition, 2012,20:265-283.

28.Ruhnau, E. The deconstruction of time and the emergence of temporality. In Time, Temporality, Now eds. Harold Atmanspacher and Eva Ruhnau, E. 53-70. Berlin: SpringerVerlag. 1997

29.Montemayor, C.; Wittmann, M. The Varieties of Presence: Hierarchical Levels of Temporal Integration. Timing \& Time Perception.2014, 2:325-338. 
30.Foundalis H. Why does time 'flow' but space is? Answers in evolution and cognition. Essay for the Fqxi contest on the nature of time. September 2, 2014, http://fqxi.org/community/forum/topic/276.

31.Arstila, V. Temporal Experiences without the Specious Present. Australasian Journal of Philosophy 2018,96:287-302.

32.Block, R. A.; Patterson. R. Simultaneity, successiveness, and temporal-order judgments. In Encyclopedia of Time ed. Samuel L. Macey,555-557.

NewYork: Garland.1994

33.Block, R. A.; Zakay, D. "Prospective and retrospective duration judgments: A meta-analytic review.” Psychonomic Bulletin Review1997,s 4:184-197.

34.Montemayor, C.. Conscious awareness and time perception. PsyCH Journal, 2017,6(3):228238.

35.Buonomano, D. V. Your brain is a time machine. London: W. W. Norton.2017

36.Droit-Volet, S,; Wearden, J.H. Passage of time judgments are not duration judgments:

Evidence from a study using Experience Sampling Methodology. Frontiers in Psychology 2016,7: 176.

37.Davies, P. How to build a time machine. Penguin 2003

38.Scholl, B. Object persistence in philosophy and psychology. Mind and Language, 2007,22:563-591

39. Velleman D. So it goes. The Winnower. 2016,16:1-16.

40.Aerts, D. Quantum theory and human perception of the macro-world. Frontiers in Psychology 24June 2014https://doi.org/10.3389/fpsyg.2014.00554.2014

41.Muller R.A. Now: the physics of time. New York, W.W. Norton.2016

42.Muller, R. A. ; Maguire, S. 'Now' and the flow of time.2016. ArXiv: 1606.07975

43.Barbour, J. ; Koslowski, T. ; Mercati. F."Janus points and arrows of time." arXiv preprint arXiv:1604.03956 (2016).

44.Barbour, Julian. "Double time." New Scientist 2021.3324, 47-49. 